\title{
Chemiluminescence Immunoassay: Basic Mechanism and Applications
}

\author{
Mohammad Anwar-ul Azim, Mizanul Hasan, Israque Hossain Ansari and Faria Nasreen \\ National Institute of Nuclear Medicine and Allied Sciences, BAEC, BSMMU, Shahbagh, Dhaka
}

Correspondence Address : Dr. Mohammad Anwar-Ul Azim, Senior Scientific Officer, In vitro Nuclear Medicine Division, National Institute of Nuclear Medicine \& Allied Sciences (NINMAS), Bangladesh Atomic Energy Commission (BAEC), E-mail: anwarri79@gmail.com

\begin{abstract}
Chemiluminescence immunoassay (CLIA) has been widely applied in different fields including environmental monitoring, liquid chromatography, clinical diagnosis; food safety, pharmaceutical analysis, immuno- and gene probe assays, as a promising approach for selective, sensitive, rapid and simple analysis. It is often necessary to detect a large number of complicated or low-abundance samples. However, traditional methods need great consumption of time, reagents and labor, which limit their clinical applications. As a result, rapid, high-throughput, sensitive and low-cost detection methods have become the development trend of CLIA. A new chemiluminescence immunoassay analyzer with advanced acridinium ester (AE) technology will be installed at the radioimmunoassy laboratory of National Institute of Nuclear Medicine and Allied Sciences (NINMAS) shortly. In conjunction with the existing Radioimmunoassay (RIA), Immunoradiometric assay (IRMA) and DissociationEnhanced Lanthanide Fluorescent Immunoassay (DELFIA), introduction of chemiluminescence immunoassay (CLIA) technology will expand the activities of in vitro immunoassay. It will also improve the research and development activities of in vitro division of NINMAS. In this review article, we have summarized the basic principle of chemiluminescence immunoassay and its applications. A comparison of RIA, CLIA and enzyme-linked immunosorbent assay (ELISA) was also briefly described in the article.
\end{abstract}

Key words: Chemiluminescence immunoassay (CLIA), Acridinium ester (AE) technology, Enzyme-linked immunosorbent assay (ELISA), Radioimmunoassay (RIA), Immunoradiometric assay (IRMA).

\section{INTRODUCTION}

Luminescence is the most conveniently defined as the radiation emitted by a molecule, or an atom, when these species return to the ground state from the exited state. According to the source of excitation, luminescence phenomenon could be classified as photoluminescence (fluorescence and phosphorescence) when the excitation source is energy from absorbed light, chemiluminescence-energy from chemical reactions and bioluminescence energy from biologically catalysed reactions (1). The phenomenon of bioluminescence occurs widely in nature and has been studied for many years. Analytical applications of bioluminescence have been comparatively recent and followed the discovery that ATP was a cofactor for the luciferase-catalysed luminescence of firefly luciferin (2) (Figure 1). This observation has led to the development of methods for quantitating ATP both as an indicator of the presence of living cells and as a product of various enzyme systems (3). More recently, there has been considerable interest in synthetic molecules, which can be oxidized to undergo chemiluminescent reactions. Eilhardt Weideman (1988) first invented the term "chemiluminescence". Chemiluminescent reaction refers to the emission of light of varying degrees of intensity and lifetime, with colors that span the visible spectrum, from a chemical transformation $(4,5)$ (Figure 2).

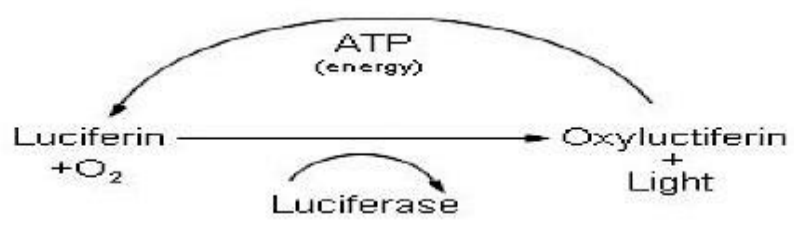

Figure 1: Bioluminescence

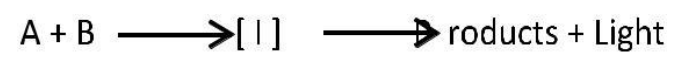

Figure 2: Chemiluminescence reactions 
Chemiluminescent uimmunoassay (CLIA) has gained increasing attention in different fields, including life science, clinical diagnosis, environmental monitoring, food safety and pharmaceutical analysis (6) because of its high sensitivity, good specificity, wide range of applications, simple equipment and wide linear range. Immunoreaction is the recognition between antigen and antibody. Because of the low diffusion rate of these large molecules and the site block problem, the immunoreaction rate controlled by mass transport and reaction kinetics is generally low. Conventional immunoassay always needs a long incubation time, which in turn causes the whole analysis to take several hours for completion, so the throughput and application range are largely limited (7). Researchers have designed different methods to shorten the analysis time by improving mass transport and reaction kinetics. The rapid immunoassay has expanded the applications of CLIA.

Traditionally, immunoassay is performed as discrete tests, i.e., one analyte per assay run, and several runs are needed to detect all the components in a complex system. Great consumption of time, reagents and labor limits the application of the traditional strategy. Since a multianalyte immunosensing system has the advantages of high throughput, short time, low consumption and low cost, it has been very popular during the recent years. It is also necessary to detect samples of low abundance, so developing highly sensitive CLIA has become a new trend. The main approach to improving the analysis sensitivity is to reduce the noise and enhance the signal. In order to reduce the noise, small proteins are always used to block the active site on the biosensing interface. Signal amplification techniques focus on constructing functionalized interface and designing novel trace tags (7).

A new chemiluminescence immunoassay analyzer (ADVIA Centaur XPT Immunoassay System, Siemens Healthcare Diagnostics) with advanced acridinium ester (AE) technology will be installed at the radioimmunoassy laboratory of National Institute of Nuclear Medicine and Allied Sciences (NINMAS) shortly, under the annual development project "Upgrading and strengthening the facilities at National Institute of Nuclear Medicine and Allied Sciences". In conjunction with the existing Radioimmunoassay (RIA), Immunoradiometric assay (IRMA) and Dissociation-Enhanced Lanthanide Fluorescent Immunoassay (DELFIA), introduction of chemiluminescence immunoassay (CLIA) technology will be the first time in in vitro nuclear medicine of NINMAS and also other Institute of Nuclear Medicine and Allied Sciences (INMAS) of Bangladesh Atomic Energy Commission (BAEC). This article summarized the basic principle of chemiluminescence immunoassay and its applications. A comparison of RIA, CLIA and enzyme-linked immunosorbent assay (ELISA) was also briefly described in the article.

\section{CHEMILUMINESCENCE IMMUNOASSAY (CLIA)}

CLIA is a method to determine the concentration of samples according to the intensity of the luminescence that the chemical reaction emits. In general, chemiluminescence reaction yields one of the reaction products in an electronic excited state producing light on falling to the ground state. As can be seen in Figure 3 , the process of light emission in chemiluminescence is the same as in photoluminescence, except for the excitation process. In fluorescence and phosphorescence, the electronically excited state is produced by absorption of UV-visible light returning to the ground state $\left(\mathrm{S}_{0}\right)$ from the lowest singlet excited state $\left(\mathrm{S}_{1}\right)$ or from the triplet excited state $(\mathrm{T} 1)(8,9)$.

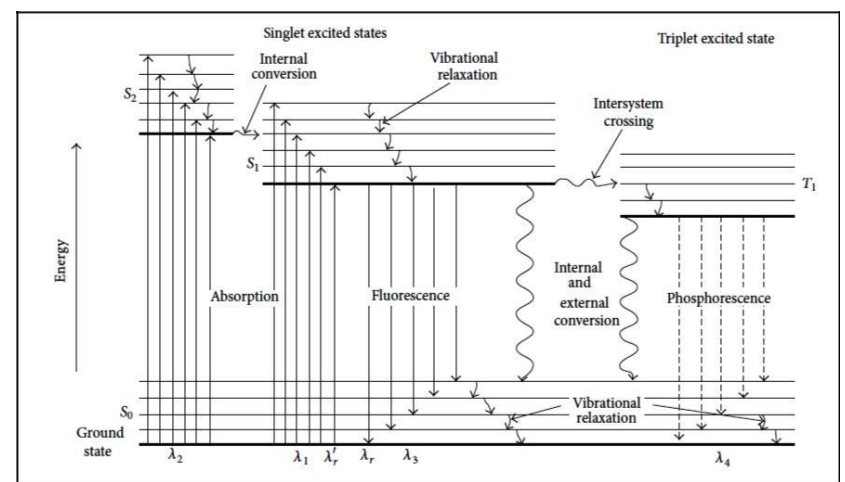

Figure 3: Jablonski Diagram describing the electronic levels of common organic molecules and possible transitions between different singlet and triplet states. (M. Sauer, J. Enderlein. Handbook of Fluorescence Spectroscopy and Imaging) 
In general, a chemiluminescent (CL) reaction can be generated by two basic mechanisms (Figure 4) in a direct reaction, two reagents, usually a substrate and an oxidant in the presence of some cofactors, react to from a product or intermediate, sometimes in the presence of a catalyst. Then some fraction of the product or intermediate will be formed in an electronically excited state, which can subsequently relax to the ground state with emission of a photon. The substrate is the CL, precursors, which is converted into the electronically excited molecule, responsible for light emission or acting as the energy as transfer donor in indirect CL. The most popular CL substrates are luminol, isoluminol and their derivatives, acridinium ester derivatives, peroxidase and alkaline phosphatase (7). On the contrary, indirect or sensitized CL is based on a process of transfer of energy of the excited specie's to a fluorophore (10). This process makes it possible for those molecules that are unable to be directly involved in $\mathrm{CL}$ reaction to transfer their excess of energy to a fluorophore that in turn is excited, releasing to its ground state with photon emission. All of these paths lead to a great variety of practical uses of CL in solid, gas, and liquid phases (11).

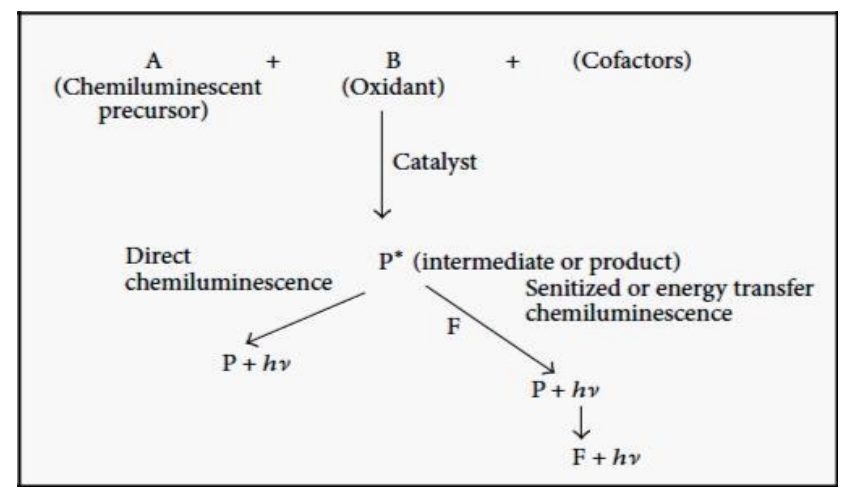

Figure 4: Types of $C L$ reaction; $P=$ Product and $f=$ fluorescing substance

Chemistry of chemiluminescent labels: The high specific activity and the sensitivity of detection of the labelling material are very important in case of chemiluminescence immunoassay reactions. Conventional enzyme labels or fluorescent derivatives have detection limits in the region of $10^{-14} \mathrm{~mol}$ and consequently have been successfully applied only to low sensitivity assay systems. However, both luminol and acridinium salts have detection limits of approximately $10^{-}$ 18 mol using available luminometers, thus matching and even surpassing the sensitivity of ${ }^{125}$ I detection (12). However, it has become apparent that the association of luminol with protein can drastically affect the quantum yield of the reaction. This is evidenced by work with derivatives of isluminol, in particular Aminobutylethyl isoluminol $(\mathrm{ABEl})$, which can be coupled to low molecular weight compounds without loss in quantum yield (13) and related molecules have been used as labels for a variety of steroid immunoassays which have levels of performance comparable to those of the corresponding radioimmunoassays (14). A similar loss in quantum yield was observed when aminohexylethyl isoluminol was used to label antibodies to hepatitis B surface antigen (12).

The use of acridinium ester derivatives (Figure 5), such as DMAE, NSP-DMAE, HEGAE (www.siemens.disgnodtics)), HQYAE (15), ZAE (16) etc., have potential advantages as compared with luminol derivatives and can be explained with the figure 6, which summarizes their light-emitting reactions. Both reactions are oxidative and occur in alkaline conditions, though in the case of luminol, there is the absolute requirement for a catalyst. This can be a simple transition metal cation such as $\mathrm{Mn}^{++}$or $\mathrm{Ni}^{++}$but may also be a complex macromolecule such as horseradish peroxidase and cytochromes (17). The most popular catalyst is microperoxidase since this yields an efficient reaction under relatively mild conditions. This requirement for a catalyst renders the reaction susceptible to interference from materials present in biological samples while the powerful oxidizing conditions may result in high background effects (12).

In contrast, the mild conditions required for acridinium ester reactions produce relatively low background chemiluminescence. Moreover, the reaction itself involves an oxidative cleavage to yield a dioxetanone intermediate before the formation of the excited product molecule $\mathrm{N}$ methyl acridone (Figure 7). As a result of the cleavage the N-methyl acridone is dissociated prior to the light emission so that quenching by associated protein is avoided (15). 


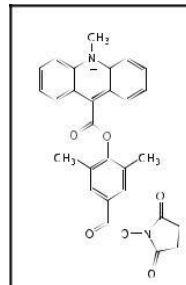

(I)

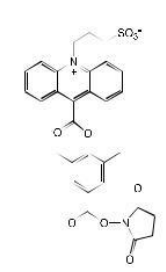

(II)

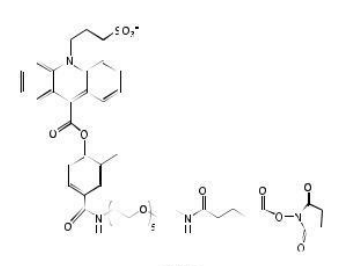

(III)

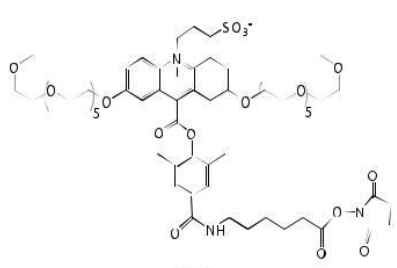

(IV)

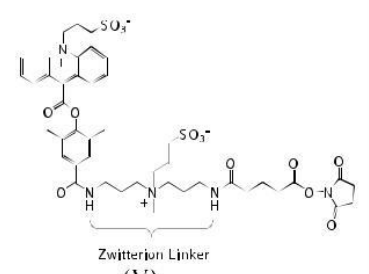

(V)
(I): Dimethyl acridinium ester (DMAL); (II): N-Sulfopropyl dimethyl acridinium ester (NSP-DMAL) (III): Hexa(ethyllencolycol) Acridinium Lister (HLG.LL)

(IV): High Quantum Yield $\Lambda$ cridinium Lster (HQYAL) with a pair of methoxy-hexa (ethylenc) glycol groups at $\mathrm{C}-2$ and or $\mathrm{C}-7$ in the acridinium ring

Figure 5: Derivatives of acridinium ester

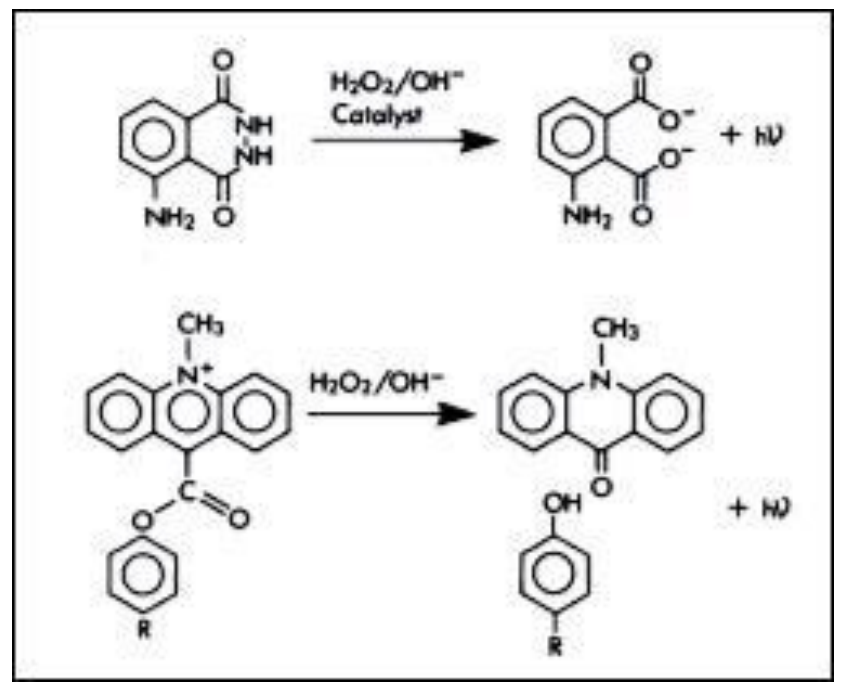

Figure 6: Chemiluminescent reaction of Luminol and acridinium ester

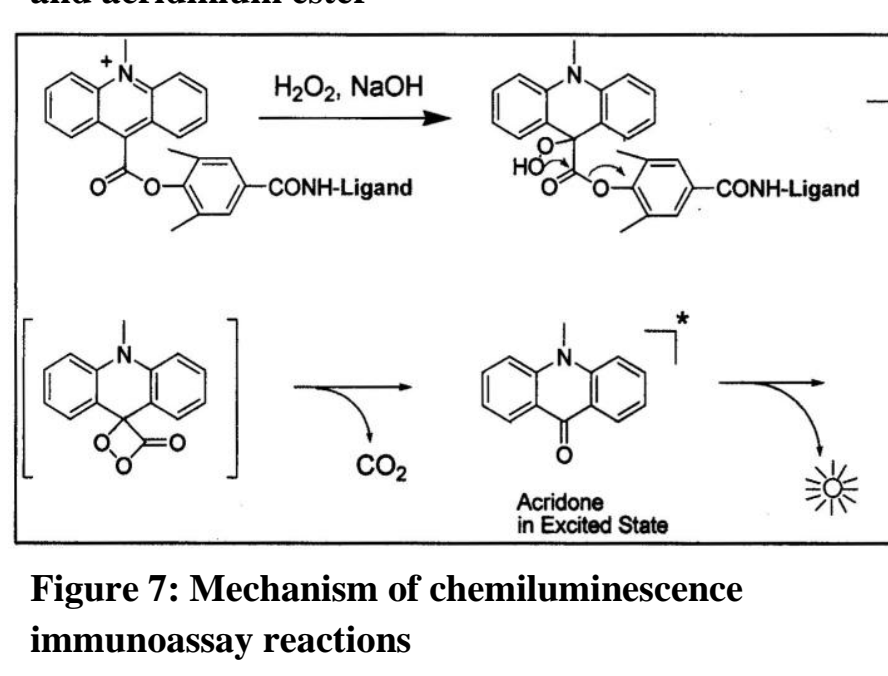

Figure 7: Mechanism of chemiluminescence immunoassay reactions

\section{CHEMILUMINESCENCE IMMUNOASSAY AND} RADIOIMMUNOASSAY

The quantitative inhibition of binding of labelled antigen to antibody by increasing concentrations of unlabeled antigen forms the basis of radioimmunoassay (RIA). Two fundamental aspects have made RIA one of the most important techniques in diagnostic and therapeutic medicine in recent years. Firstly, the specificity of the immune reaction means that analysis can be carried out in complex mixtures such as serum. Secondly, the realisation that antibodies can be produced not only to high molecular weight proteins but also low molecular weight compounds such as drugs and steroids provided they are coupled to a suitable carrier.

IRMA is an alternative procedure to RIA, which used the same type of immunological reaction but was based on a different principle. RIA technique involves competition between labeled antigen and unlabeled antigen (as standards or unknowns) for a limited number of antibody binding sites, whereas, IRMA relies on the conversion of antigen to a labelled derivative by reaction with labeled antibody. The derivative is then measured directly. The main advantage of IRMA is that performance is optimal when excess-labeled antibody is used, the consequence being that all antigen is rapidly derivatized. These rapid reaction kinetics contrasts with those seen in RIA where high sensitivity is achieved by diluting out the antibody with the result that extended reaction times may be required to provide the desired sensitivity. Until recently both RIA and IRMA have relied on the use of ${ }^{125}$ I as the label. This isotope is a moderately high-energy $\gamma$-emitter with a half-life of 60 days and its sensitivity of detection is excellent, approximately $5 \mathrm{x}$ $10^{-18} \operatorname{mol}(12)$.

A unique advantage of radioactive labels is that quantitation is unaffected by their environment. This is not true in the case of non-isotopic compounds. Luminescent molecules are susceptible to quenching of the signal by constituents of biological fluids

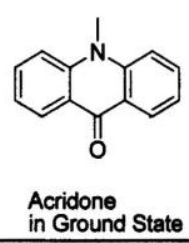

such as serum. In general, this problem has been avoided by the use of solid - phase antibody technology. As a result, the 
antibody bound fraction can be isolated from and washed free of potentially interfering compounds before signal detection.

CLIA offers distinct advantages over RIA. First and foremost, they eliminate the use of radioactive labels and the concomitant concerns regarding exposure of personnel to radiation and waste disposal. In addition, these assays use reagents with a longer shelf life, a higher sensitivity, and a shorter running time (18). The estradiol RIA, for example, uses estradiol labeled with $125 \mathrm{I}$ and has a shelf life of only 2 months because of the short half-life of ${ }^{125}$ I.

Also, iodinated steroids tend to have a higher binding affinity for the antibody than the natural steroid, thus decreasing the detection sensitivity of the assay. While RIA requires 1-3 hours incubation time and then 1 minute per tube for quantification, a CLIA using acridinium esterlabeled estradiol requires only 30 minutes for incubation and only 5 seconds for quantification (18). Avner Hershlag et al 2000 (18) reported that in case of patients receiving gonadotropins for ovarian stimulation, CLIA results for estradiol correlated closely with RIA results. However, in patients receiving oral estradiol to prepare the endometrium for transfer of frozen embryos, marked discordance was observed between CLIA and RIA values of estradiol.

A two-site immunochemiluminometric assay (ICMA) methodology (Figure 8) to yield highly sensitive assay for human thyroid stimulating hormone (TSH) is at present under evaluation (19). In this assay, the analyte is reacted either sequentially or simultaneously with labeled antibodies and solid-phase antibodies. In this procedure, the uptake of labelled antibody on to solid-phase antibody is a function of the quantity of analyte bound and hence the analyte concentration in the sample.

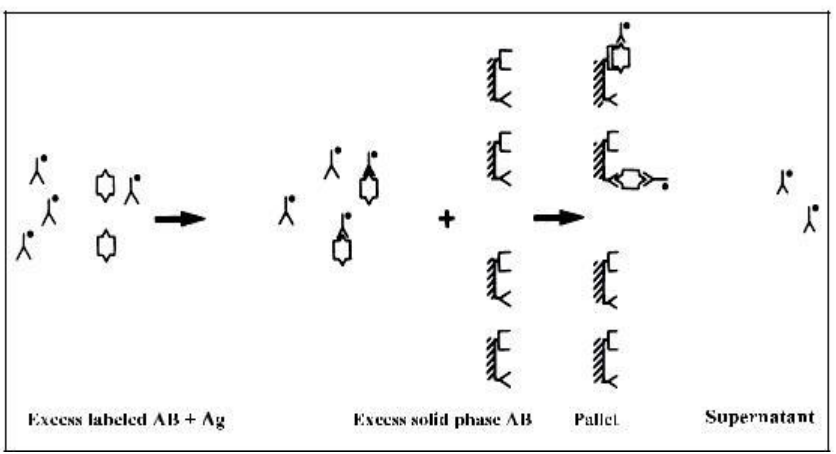

Figure 7: Two-site immunochemiluminometric assay (ICMA) of human thyrotropin (TSH)
In this procedure standards or serum samples $(100 \mu \mathrm{L})$ are reacted with a monoclonal antibody $(100 \mu \mathrm{L}, 1.5$ ng) labelled with $3 \mathrm{~mol} / \mathrm{mol}$ acridinium ester. After $2 \mathrm{~h}$, solid-phase antibody suspension $(100 \mu \mathrm{L}, 50 \mu \mathrm{g})$ is added and after a further $1 \mathrm{~h}, 1 \mathrm{~mL}$ of buffer is added and the mixture centrifuged. After one further $1 \mathrm{~mL}$ wash, the chemiluminescent activity associated with the solid-phase is quantified luminometrically following injection of $200 \mu \mathrm{L}$ water to resuspend the pellet followed by $200 \mu \mathrm{L} 0.1 \%$ (v/v) $\mathrm{H}_{2} \mathrm{O}_{2}$ in $0.1 \mathrm{M} \mathrm{NaOH}$. The photon counts are integrated over a $2 \mathrm{~s}$ period and the bound counts related to the dose of analyte present. The use of reagent excess methodology together with an acridinium ester label of high specific activity combines to make this the most sensitive immunoassay yet described for TSH (20).

Zhang Ming et al 2014 (21) compared the results of anti-thyroglobulin antibody (anti-TgAb) and antithyroid peroxidase antibodies (anti-TPOAb), detected by CLIA and RIA. It was reported that the serum level of anti-TgAb and Anti-TPOAb in patients and control group tested by CLIA and RIA had no statistically significant differences.

Rojanasakul A et al 1994 (22) assayed the blood samples from eight volunteers during one menstrual cycle for estradiol, progesterone, luteinizing hormone (LH), follicle stimulating hormone ( $\mathrm{FSH}$ ) and prolactin by chemiluminescence immunoassay (CLIA) and radioimmunoassay (RIA). Comparison of the results revealed that CLIA yielded lower mean values of $\mathrm{LH}$, FSH, progesterone, but higher mean values of prolactin than RIA. Mean estradiol levels assayed by the two methods were reported similar. There was a good correlation between the hormonal values performed by the two methods. By calculation, the CLIA hormonal value could accurately predict the RIA value by 96.6 , 93.9, 89.9 and $66.0 \%$ for progesterone, LH, FSH and prolactin, respectively.

Chen CK et al 1996 (23) evaluated the performance of chemiluminescent immunoassay (CLIA) system (Immulite, Diagnostic Products Corp, Los Angeles, CA, USA) and compared it with a popular 
Immunoradiometric immunoassay (IRMA) system. In that study, the data of 158 patients whose thyroid function was assessed and that of another 158 patients whose parathyroid function was assessed were analyzed. The thyroid-stimulating hormone (TSH) CLIA is an ultrasensitive TSH (us-TSH) assay that provided more detailed, reliable results for low TSH concentrations than TSH IRMA. Coupled with the free-T4 assay, the us-TSH assay enhanced the ability to detect and monitor the status of thyroid dysfunction. Intact-parathyroid hormone (PTH) CLIA detected lower serum intact-PTH levels, was found more sensitive and as reliable as IRMA.

Jing Hua et al 2005 (24) compared the characteristics of chemiluminescent immunoassay (CLIA), radioimmunoassay (RIA) and magnetic solid phase enzyme-linked immnosorbent assay (MSP-ELISA) in measuring thyroid hormones, total T3, total T4, FT3, FT4 and TSH in human serum and standard samples of thyroid hormones by CLIA, RIA and MSP-ELISA respectively. No statistical differences between CLIA, RIA and MSP-ELISA in linearity and relativity were reported. CLIA was better than RIA and MSP-ELISA in precision and accuracy.

The concentration of carcinoembryonic antigen (CEA) in serum obtained from different carcinosis patients was detected by using the method of double antibody sandwich CL immunoassay and the results obtained by this method are fairly well agreeable to those obtained by RIA method (25). The $\alpha$-fetoprotein (AFP) is the most widely used tumor marker, consistently can be measured through CLIA for the diagnosis of Hepatocellular carcinoma $(26,27)$.

Prostate cancer ( $\mathrm{PCa}$ ) has become a most widespread and stubborn disease and a major cause of death in the old age male population nowadays. Zheng $\mathrm{Y}$ et al 2008 (28) development sensitive chemiluminescence immunosensor for the detection of PSA. Most of the current PSA detection methods are usually based on immunoassays and CLIA is among the most widely used readout modality in virtue of undoubted advantages over other more widely used systems including enzyme-linked immunosorbent assays
(ELISA), time-resolved immunofluorometric assay, surface plasmon fluorescence immunoassay, bioluminescent immunoassay, electrochemical and surface enhanced Raman scattering (SERS) (29).

\section{ENZYME-LINKED IMMUNOSORBENT ASSAY (ELISA) AND CLIA}

The enzyme-linked immunosorbent assay (ELISA) is a popular type analytic biochemistry assay that uses a solidphase enzyme immunoassay (EIA) to detect the presence of a substance, usually an antigen, in a liquid sample or wet sample. In ELISA, antigens from the sample are attached to a surface. Then, a further specific antibody is applied over the surface so it can bind to the antigen. This antibody is linked to an enzyme, and, in the final step, a substance containing the enzyme's substrate is added. The subsequent reaction produces a detectable signal, most commonly a color change in the substrate. ELISA technology is derived from RIA, but with limited exposure to radiation risk.

Due to its advantage in sensitivity, speed, and automation, chemiluminescence immunoassay (CLIA) has played a key role in promoting development of detecting ultra micro active substances in clinical diagnosis and prognosis. Nowadays, as a rapidly increasing technology in the immunology market, CLIA has become one of the most important members in in vitro diagnosis (IVD) medical devices.

Sometimes, it is quite difficult to detect the concentration of some indicators by ELISA due to its low concentration in samples, such as human chorionic gonadotropin (hcG). However, the CLIA Kit for Chorionic Gonadotropin (CG) could be utilized in the detection because that its minimum detection range levels at $2.35 \mathrm{pg} / \mathrm{ml}(30)$.

Characteristic comparison of CLIA and ELISA kit: Chemiluminescence Kit (CLIA Kit) is a kind of sensitive Kit, which uses Streptavidin-horseradish peroxidase (HRP) as enzyme and enhanced ECL chemiluminescent system as substrate reagent (30). The characteristic comparison of the CLIA and ELISA kit is illustrated in Table 1 
Table 1: The characteristic comparison of the CLIA and ELISA kit (30).

\begin{tabular}{|l|l|l|}
\hline \multicolumn{1}{|c|}{ Characteristic } & \multicolumn{1}{|c|}{ CLIA Kit } & \multicolumn{1}{c|}{ ELISA Kit } \\
\hline High sensitivity & $\begin{array}{l}\text { Minimum detection range levels at } \\
10-18 \mathrm{~mol} / \mathrm{L} \text {, important implication for } \\
\text { early diagnosis of diseases }\end{array}$ & $\begin{array}{l}\text { Minimum detection range levels } \\
\text { at 10-9 mol/L }\end{array}$ \\
\hline linear range & $\begin{array}{l}\text { RLU values show a linear relationship } \\
\text { within 4-6 orders of magnitude }\end{array}$ & $\begin{array}{l}\text { Optical density(OD) values show } \\
\text { a linear relationship within 2 } \\
\text { orders of magnitude }\end{array}$ \\
\hline Sample dilution & $\begin{array}{l}\text { Sample with high concentration could } \\
\text { be detected with stock sample to avoid } \\
\text { the deviation in dilution }\end{array}$ & $\begin{array}{l}\text { Sample with high concentration } \\
\text { should be diluted before } \\
\text { detection, and this might cause } \\
\text { some deviation }\end{array}$ \\
\hline $\begin{array}{l}\text { Dilution fold of } \\
\text { standard curve }\end{array}$ & $\begin{array}{l}\text { 3-4 fold dilutions series, the range of } \\
\text { standard curve is quite wide, and the } \\
\text { highest detectable concentration is } \\
10000 \text { times higher than the lowest } \\
\text { detectable concentration }\end{array}$ & $\begin{array}{l}2 \text { fold dilutions series, and the } \\
\text { highest detectable concentration } \\
\text { is 128 times higher than the } \\
\text { lowest detectable concentration }\end{array}$ \\
\hline Sample volume & $\begin{array}{l}50 \text { ul of sample was utilized in some } \\
\text { CLIA Kit }\end{array}$ & $\begin{array}{l}100 \text { ul of sample was utilized in } \\
\text { regular sandwich ELISA Kit }\end{array}$ \\
\hline
\end{tabular}

\section{PERSPECTIVE AND FUTURE}

CLIA has been widely applied in different fields including environmental monitoring, liquid chromatography, clinical diagnosis; food safety, pharmaceutical analysis, immunoand gene probe assays. Nowadays, the CLIA represents a potent and versatile medical analytical tool suitable for a wide range of applications, because it combines the high delectability of the of the luminescence signal with the possibility to localize and quantify the light emission in a sample.

The high detectability and rapidity of CL techniques, along with the availability of microarray-based analytical devices, allows the development of high throughput screening assays, in which simultaneous, multi-analyte detection is performed on multi sample. Therefore, more and more medical experts and chemists are interested in CLIA. However, the development of CLIA is dependent on the application of the sensitive and selective chemiluminescent probe. Improvements in analytical sensitivity will likely lead to the discovery of new analytes tumours detection. Technical enhancement holds the promise of detecting very low concentrations in serum using nanoparticles as labels and CL detection $(31,32)$.

\section{CONCLUSION}

Chemiluminescence immunoassay offers a viable and logical alternative to a range of analytical immunoassay based on the use of radioisotopes. Its feasibility has been demonstrated for a range of analytes including both high and low molecular weight species. The stability of the reagents and their analytical performance has important implications in improving and maintaining high quality assay performance. Most importantly the development of ICMA technology based on labelled antibodies has the potential for increasing assay sensitivity. It is likely that this technology will prove valuable in the detection and monitoring of infective agents as well as tumour markers where conventional immunoassay technology provides insufficient sensitivity.

Introduction of new chemiluminescence immunoassay system at NINMAS, along with the gold standard radioimmunoassay method will be great scope for doing comparative study of the in vitro immunoassay results, measured by CLIA and RIA. It will definitely increase the test range and also speed up the patient's services of in vitro division. Most importantly, installation CLIA will heighten the research scope and activities of in vitro division of NINMAS.

\section{REFERNCES}

1. Tadesse Haile Fereja, Ariaya, Thirumurugan Gunasekaram, A recent review on chemiluminescence reaction, principle and application on pharmaceutical analysis. Hindawi Publishing Corporation, ISRN Spectroscopy, Volume 2013, Article ID 230858, 12 pages.

2. A. Lundin, In 'Clinical \& Biochemical Luminescence' L.J. Kricka and T.J.N. Carter (eds), Marcel Dekker, New York, 1982; 44-74.

3. R.C. Allen and L.D. Loose, Biochem. Biohys. Res. Comm. 1976; 69, 245-252.

4. Carlson R, Lewis SW, Lim KF, Seeing the light: using chemiluminescence to demonstrate chemical fundamentals. Aust J Chem Educ, 200; 14: 51-53.

5. Xiao Q, Li H, Lin JM, Development of a highly sensitive magnetic particle-based chemiluminescence enzyme immunoassay for thyroid stimulating hormone and comparison with two other immunoassays. Clinica Chimica Acta, 2010; 411 : 1151-1153.

6. Lin J M, Zhao L X, Wang X. Chemiluminescence 
Immunoassay. Beijing: Chemical Engineering Press, 2008; 4-6.

7. WANG Chen, WU Jie, ZONG Chen, XU Jie, JU HuangXian, Chemiluminescent immunoassay reactions and applications. Chinese Journal of analytical Chemistry, 2012; 40 (1), 3-10.

8. J. Hodak, D. Harvey, and B. Valeur, Introduction to Fluorescence and Phosphorescence, 2008.

9. E. L. Wehry, Molecular fluorescence, phosphorescence, and chemiluminescence spectrometry. Analytical Chemistry, 1980; vol. 52, no. 5.

10. M. Sauer, J.Hofkens, and J. Enderlein, Handbook of Fluorescence Spectroscopy and Imaging. WILEY-VCH Verlag GmbH \& Co. KGaA,Weinheim, Germany, 2011.

11. J. Han, J. Jose, E. Mei, and K. Burgess, Chemiluminescenenergy-transfer cassettes based on fluorescein and nile red. Angewandte Chemie International Edition, 2007; vol. 46, no. 10, 1684-1687.

12. J. Stuart Woodhead and Ian Weeks, Chemiluminescence immunoassay. Pure \& appl. Chem., 1985; vol.57, No. 3, 523-529.

13. H.R. Schroeder, R.C. Boguslaski, R.J. Carrico, et al., In 'Methods in Enzymology'. Vol. 57, M.A. DeLuca and W.D. McElroy (eds.), Academic Press, New York, 1978; 424-455.

14. W.P. Collins, G.J. Barnard, J.B. Kim, et al. In 'Immunoassay for Clinical Chemistry', 2nd ed., W.M. Hunter and J.E.T. Corrie (eds.), Churchill Livingstone, Edinburgh, 1983; 373-397.

15. Natrajan, D. Sharpe and D. Wen, "Effect of Surfactants on the Chemiluminescence of Acridinium Dimethylphenyl Ester Labels and their Conjugates", Org. Biomol. Chem., 2011; 9(14), 5092-5103.

16. A. Natrajan, D. Sharpe, J. Costello and Q. Jiang, "Enhanced Immunoassay Sensitivity Using Chemiluminescent Acridinium Esters with Increased Light Output”, Analytical Biochemistry, 2010;406, 204-213.

17. H.R. Schroeder and F.M. Yeager, Anal. Chem. 1978; 50, 1114-1120.

18. Avner Hershlag, Michael Zinger, Martin Lesser, Gerald Scholl, Loring Bjornson, Is chemiluminescence immunoassay an appropriate substitution for radioimmunoasaay in monitoring estradiol levels? Fertility and sterility. June 2000; Vol.73, No. 6, 1174-1178.

19. E. Bolton andM.M. Richter, "Chemiluminescence of Tris(2,2'-bipyridyl) ruthenium (II): a glowing experience,” Journal of Chemical Education, 2001; vol. 78, no. 1, 4748.
20. W. P. Collins, Complementary Immunoassays, John Wiley \& Sons, 1988.

21. Zhang Ming, Wang Xi-qing, Wang Jin, Comparison of Chemiluminescence immunoassay and Radioimmunoassay for the detection of Serum Antithyroglobulin antibodies and Anti-thyroid Peroxidase antibodies. Labeled Immunoassay \& Clin Med, 2014; Vol. 21, No.6, 722-725.

22. Rojanasakul A, Udomsubpayakul U, Chinsomboon S, Chemiluminescence immunoassay versus radioimmunoassy for the measurement of reproductive hormones. Int J Gynaecol Obstet. May 1994; 45 (2), 141-6.

23. Chen CK, Tsal KS, Clinical application of chemiluminescent immunoassay for thyroid stimulating hormone, free $\mathrm{T} 4$ and intact-parathyroid hormone. J Formos Med Assoc, March 1996; 95 (3), 197-202.

24. Jing Hua, Li Dan, Chen Yiguang, Zhou Huiqin, Xu Liyan, The evaluation of CLIA, RIA and MSP-ELISA for measurement of thyroid hormones. Labeled Immunoassay and Clinical Medicine, March 2005; 12 (1), 38-40.

25. Zhuang J, Huang JL, Chen GN, Synthesis of a new biacridine and its use as the chemiluminescent probe for immunoassay of carcinoembryonic antigen, Anal Chim Acta, 2004; 512: 347-353.

26. Koteish A, Thuluvath PJ, Screening for hepatocellular carcinoma. J Vasc Interv Radiol, 2002; 13: S185-190.

27. Zhou L, Liu J, Luo F, Serum tumor markers for detection of hepatocellular carcinoma. World J Gastroenterol, 2006;12: 1175-81.

28. Zheng Y, Chen H, Liu XP, Jiang JH, Luo Y, Shen GL, Yu $\mathrm{RQ}$, An ultrasensitive chemiluminescence immunosensor for PSA based on the enzyme encapsulated liposome. Talanta, 2008; 77: 809-814.

29. J. L. S. ARAÙJO, M. R. Melo-Junior, L.B. Carvalho JR, Potential applications of the Chemiluminescent methods in tumoral disease investigation. International Journal of Pharma and Bio-science, 2011; Vol 2, Issue. 2, 392-400.

30. "Chemiluminescent Immunoassay kits, higher sensitivity, wider dynamic range and lower consumption" (www. Cloud-Clone.us).

31. Hu D, Han H, Zhou R, Dong F, Bei W, Jia F, Chen H, Gold(III) enhanced chemiluminescence immunoassay for detection of antibody against ApxIV of Actinobacillus pleuropneumoniae. Analyst, 2008; 133: 768-773.

32. Jie GH, Huang $\mathrm{X}$, Sun J, Zhu J, Electrochemiluminescence of CdSe quantum dots for immunosensing of humanprealbumin. Biosens Bioelectron, 2008; 23 (12): 1896-1899. 